HEART FAILURE AND CARDIOMYOPATHY

\title{
Prognosis and prognostic factors in patients with hypertrophic cardiomyopathy in Japan: results from a nationwide study
}

\author{
Ali Nasermoaddeli, Katsuyuki Miura, Akira Matsumori, Yoshiyuki Soyama, Yuko Morikawa, Akira \\ Kitabatake, Yutaka Inaba, Hideaki Nakagawa
}

Heart 2007;93:711-715. doi: 10.1136/hrt.2006.095232

See end of article for
authors' affiliations
$\ldots \ldots \ldots \ldots \ldots \ldots . \ldots . \ldots . .$.
Correspondence to:
Dr K Miura, Department of
Epidemiology and Public
Health, Kanazawa Medical
University, 1-1 Daigaku,
Uchinada, Ishikawa 920-
0293, Japan; miura@
kanazawa-med.ac.jp
Accepted
26 September 2006
Published Online First
3 November 2006
........................

\begin{abstract}
Objective: To investigate prognosis and prognostic factors in patients with hypertrophic cardiomyopathy (HCM) in Japan.

Design: A nationwide epidemiological study.

Setting: Hospitals selected randomly from among all hospitals in Japan.

Patients: Clinical and epidemiological information for 2155 patients with HCM were collected in 1999.

Main outcome measures: Patients were classified on the basis of baseline prognostic factors. Survival rates up to 5 years were calculated by Cox's proportional hazard model for 1605 patients.

Results: During the follow-up period, 241 deaths were recorded. The crude 5-year survival rate for the entire cohort was $86 \%$ (95\% Cl 84 to 88 ), and annual mortality ranged from $2.2 \%$ to $3.0 \%$. A higher cardiothoracic ratio on chest $x$ ray (HR $1.61 ; 95 \% \mathrm{Cl} 1.26$ to 2.05 , with 1 SD $(6.2 \%)$ increase), a lower left ventricular ejection fraction (HR 1.42; $95 \% \mathrm{Cl} 1.20$ to 1.69 , with 1 SD (13\%) decrease) and the presence of left bundle branch block (HR 3.14; $95 \% \mathrm{Cl} 1.28$ to 7.71 ) were independently associated with a poorer prognosis, whereas the presence of apical hypertrophy at baseline (HR $0.58 ; 95 \% \mathrm{Cl} 0.36$ to 0.92 ) predicted a better chance of survival.

Conclusions: The nationwide survey of patients with hypertrophic cardiomyopathy yielded important information on its prognosis and prognostic factors. These observations afford, for the first time, a measure of risk stratification in patients with HCM in Japan.
\end{abstract}

$\mathrm{H}$ ypertrophic cardiomyopathy (HCM) is a relatively common cardiac disease that has been the subject of intense investigation over the past few decades, especially in Western populations. ${ }^{1-5}$ By contrast, few large-scale and prospective studies have been conducted to examine the prognosis and prognostic factors of HCM in the far-east Asian populations. Asian patients may differ considerably from Western patients in the pattern of hypertrophy distribution and clinical manifestations. ${ }^{16}$ Because of marked heterogeneity in clinical expression, it is necessary to identify the prognostic factors and their association with death, within the broad disease spectrum of HCM, to obtain a realistic clinical perspective in the far-east Asians.

In 1999, the Japanese research committees on epidemiology of intractable diseases undertook a nationwide epidemiological survey of idiopathic cardiomyopathy in Japan to describe the detailed clinicoepidemiological features for appropriate health service planning. A detailed description of the clinicoepidemiological features of patient characteristics have been presented elsewhere. ${ }^{7}$ The estimated total number of patients with HCM was 21900 (95\% confidence interval (CI) 20600 to 23 200), with a crude prevalence rate of 17.3/100 000. ${ }^{8}$ The purpose of this study was to evaluate the 5 -year survival rate according to the presence and/or level of baseline prognostic factors from this nationwide study on patients with HCM in Japan, and to clarify factors that can predict the prognosis of this disease independently and effectively.

\section{METHODS}

The nationwide survey on cardiomyopathies, including HCM, was designed to show the prevalence and clinical features of HCM in Japan. Detailed methods have been described elsewhere. $^{78}$ The Japanese Research Committee on idiopathic cardiomyopathies prepared classification criteria, which were on the basis of the report of the World Health Organization/ International Society and Federation of Cardiology task force on the definition and classification of cardiomyopathies. ${ }^{9}$ HCM was characterised by disproportionate hypertrophy of the left ventricle and occasionally also of the right ventricle, which typically involves the septum more than the free wall, but occasionally is concentric. Specific heart muscle disease, defined as heart muscle disease of known aetiology or associated with disorders of other systems, was excluded from the survey. The hospitals included in each survey were randomly selected by stratified sampling of all departments of internal medicine, cardiovascular medicine and paediatrics throughout Japan, identified in a directory of names, department addresses and number of hospital beds obtained from the Ministry of Health and Welfare in Japan.

\section{Data acquisition}

The survey investigated patients with HCM as either inpatients or outpatients in the randomly selected departments in 1998. Firstly, the questionnaire for the survey on the number of patients with HCM was mailed directly to 2414 departments in January 1999. Of those 2414 departments, 1409 (58.4\%) departments responded, reporting data on 7262 patients. The second survey was performed to collect detailed clinical data. From a total of 577 departments that reported one or more patients with HCM in the first survey, 235 departments agreed to participate in the second survey and detailed clinical data were collected from a total of 2155 patients. Patients who died before 1998 or those visiting a hospital for the first time after

Abbreviations: $A F$, atrial fibrillation; $B M I$, body mass index; $H C M$, hypertrophic cardiomyopathy; IVS, interventricular septum; LBBB, left bundle branch block; LVEF, left ventricular ejection fraction; LV, left ventricular; NYHA, New York Heart Association 


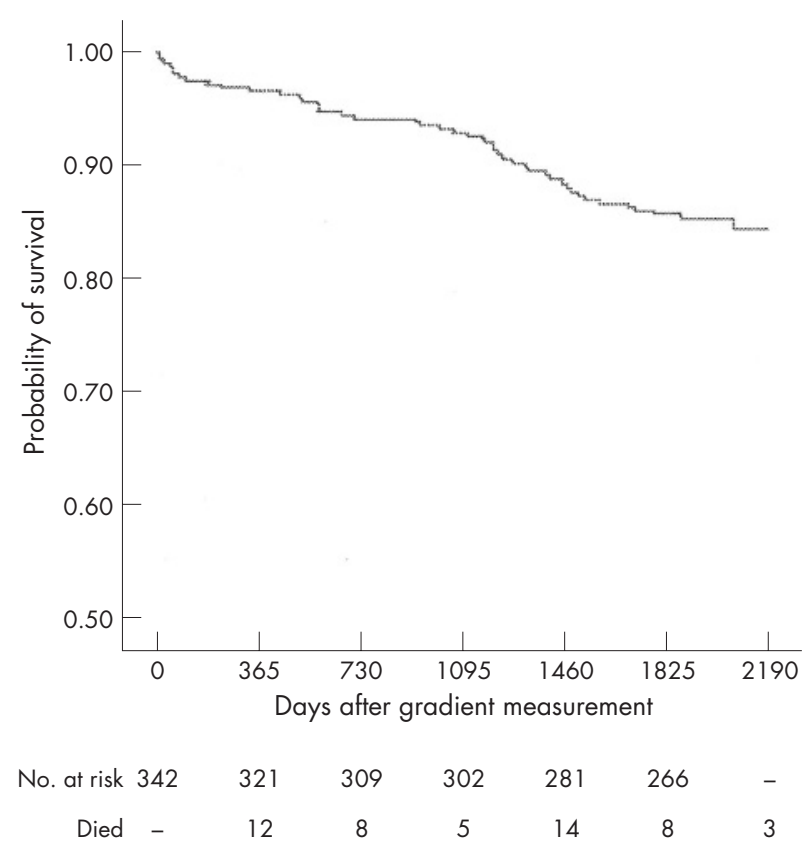

Figure 1 Kaplan-Meier survival curve of 342 patients with hypertrophic cardiomyopathy after first diagnosis in 1998.

1999 were excluded from this study as inappropriate cases, as were patients whose data were reported from more than one department (duplicate cases). The questionnaire requested detailed clinicoepidemiological information for each patient, including age, sex, symptoms and New York Heart Association (NYHA) functional class. Data from a physical examination and baseline laboratory measurements, including standard 12-lead ECG, chest $x$ ray and echocardiography (for measuring left ventricular ejection fraction (LVEF), thickness of interventricular septum (IVS), and left ventricular (LV) shape), were available. LV hypertrophy by ECG was determined by left high voltage (Minnesota code: 3-1 or 3-3). M mode echocardiographic assessment for LVEF and two dimensional echocardiographic assessment for maximum IVS thickness and LV shape were conducted for nearly all patients. Apical hypertrophy was defined as LV wall thickness confined to the most distal region at the apex below the papillary muscle level. Data on blood tests and cardiac catheterisation were only obtained for a small portion of the subjects and, therefore, we did not include them in our analysis.

\section{Medication}

Patients were generally given medical treatment as reported previously from this study. ${ }^{7}$ Medical treatment was directed toward control of symptoms, arrhythmias, coexisting hypertension and prevention of embolisation.

\section{Follow-up}

Of 235 departments reporting 2155 patients, 182 departments (reporting 1693 patients) agreed to participate in the 5-year follow-up survey. Patients' vital status was reported by doctors, with vital status for 607 withdrawn cases obtained from the residence-based register of the local government for each patient. However, follow-up was not possible for 88 patients, so these patients were excluded. Therefore, the 5-year follow-up was completed for $1605(74.5 \%)$ patients. With regard to follow-up bias, we found no significant difference for sex, age, body mass index (BMI) and NYHA functional class distribution between those who participated and those who did not participate in the follow-up. The ethical committees of the Kanazawa Medical University and the Kyoto University Graduate School of Medicine approved the study protocol.

\section{Statistical analysis}

Survival estimates were calculated using the Kaplan-Meier method, and the 5-year survival probability was calculated for the overall cohort. Patients were classified on the basis of baseline prognostic factors. The significant differences in survival rates among classifications were tested by the log rank test for trends. Hazard ratios (HRs) according to baseline characteristics were calculated by Cox's proportional hazard model, with $95 \%$ CI up to the longest follow-up time of 2190 days. The model includes variables with a $\mathrm{p}$ value $<0.05$ by the log rank test for the 5-year survival and some other important variables. HRs for continuous variables were reported for I SD change. The log minus log plotted against survival time for each covariate did not show any deviation from the proportionality assumption. The data were analysed with SPSS V.12.0J. All reported significance levels are $\mathrm{p}<0.05$ (two tailed tests).

\section{RESULTS}

Of the 1605 patients identified at baseline, 241 (15\%) died during the follow-up period. The probability of actuarial survival was calculated from the time of the baseline survey for 342 patients who were initially diagnosed in 1998 (fig 1). The crude 5-year survival rate for those diagnosed in 1998 was $86 \%$ (95\% CI 82 to 90 ). The crude 5-year survival rate for the whole cohort was $86 \%$ (95\% CI 84 to 88 ).

Table 1 shows the baseline characteristics of the patients. Of the 1605 patients in the study, 30.3\% were women and $57.1 \%$ were aged $>60$ years. Most patients (94.5\%) had experienced none or only mild symptoms (NYHA function classes I or II) at baseline.

Clinical, echocardiographic and standard 12-lead ECG variables were examined for an association with survival during the follow-up period (table 1). Crude 5-year survival rates significantly decreased with decreasing BMI, higher grade of NYHA classification, presence of atrial fibrillation (AF) or flutter, presence of left bundle branch block (LBBB), increasing CTR, decreasing LVEF and decreasing number of hospital beds. The presence of apical hypertrophy was associated with a better survival rate. There was no significant difference in the crude survival rate between men and women. Thickness of IVS, family history of HCM, the presence or absence of hypertension, and smoking and drinking habits were not associated with the 5-year survival rates.

Table 2 presents the results of proportional hazard analysis. The model includes nine variables, which were statistically significant in table 1 (age, BMI, diabetes mellitus, NYHA classification, rhythm, LBBB, apical hypertrophy, CTR and LVEF), and two other important variables (sex and IVS thickness). The multivariate-adjusted HR for all-cause mortality was not significantly different between men and women. The presence of LBBB was an independent predictor of death $(\mathrm{HR}=3.14)$, and the presence of apical hypertrophy, seen in $41.1 \%$ of study patients, resulted in a better prognosis $(\mathrm{HR}=0.58)$. The multivariate-adjusted HR for death significantly and independently increased with a $1(6.2 \%)$ SD increase of CTR, and with a 1 (13\%) SD decrease of LVEF. The Wald statistic showed that these two factors had the strongest relationship with prognosis of all the factors in the model. BMI and IVS thickness were not independently related to prognosis. A backward elimination stepwise analysis to check if there is confounding among the non-significant factors, did not find a significant difference with the results in the original model. 
Table 1 Five-year survival rates for predictive variables in patients with hypertrophic cardiomyopathy in Japan

\begin{tabular}{|c|c|c|c|c|}
\hline & n (\%) & Deaths & 5-year survival rate & $p$ Value for log rank test \\
\hline \multicolumn{5}{|l|}{ Sex } \\
\hline Men & $1118(69.7)$ & 142 & 0.87 & \\
\hline Women & $487(30.3)$ & 85 & 0.84 & 0.151 \\
\hline \multicolumn{5}{|l|}{ Age (years) } \\
\hline$<30$ & $157(9.9)$ & 14 & 0.90 & \\
\hline $30-59$ & $531(33.1)$ & 42 & 0.92 & \\
\hline$\geqslant 60$ & $917(57.1)$ & 158 & 0.82 & $<0.001$ \\
\hline \multicolumn{5}{|l|}{ BMI $\left(\mathrm{kg} / \mathrm{m}^{2}\right)$} \\
\hline$<20$ & $182(14.0)$ & 40 & 0.77 & \\
\hline $20-24.9$ & $733(56.3)$ & 92 & 0.87 & \\
\hline$\geqslant 25$ & $387(29.7)$ & 39 & 0.90 & $<0.001$ \\
\hline \multicolumn{5}{|l|}{ Family history of HCM } \\
\hline Yes & 207 (16.9) & 27 & 0.87 & \\
\hline No & $1015(83.1)$ & 132 & 0.87 & 0.991 \\
\hline \multicolumn{5}{|l|}{ Hypertension } \\
\hline Yes & $456(31.2)$ & 68 & 0.85 & \\
\hline No & $1005(68.8)$ & 129 & 0.87 & 0.336 \\
\hline \multicolumn{5}{|l|}{ Diabetes mellitus } \\
\hline Yes & $131(8.9)$ & 30 & 0.76 & \\
\hline No & $1332(91.1)$ & 178 & 0.86 & 0.002 \\
\hline \multicolumn{5}{|l|}{ Alcohol drinking } \\
\hline Yes & $475(36.3)$ & 71 & 0.85 & \\
\hline No & $832(63.7)$ & 113 & 0.86 & 0.525 \\
\hline \multicolumn{5}{|l|}{ Smoking } \\
\hline Yes & $535(39.3)$ & 68 & 0.87 & \\
\hline No & $825(60.7)$ & 115 & 0.86 & 0.524 \\
\hline \multicolumn{5}{|l|}{ NYHA classification } \\
\hline 1 & 908 (65.3) & 89 & 0.90 & \\
\hline ॥ & $406(29.2)$ & 62 & 0.84 & \\
\hline IIII & $62(4.5)$ & 28 & 0.53 & \\
\hline IV & $14(1.0)$ & 7 & 0.43 & $<0.001$ \\
\hline Rhythm & & & & \\
\hline Sinus rhythm & $1347(91.4)$ & 161 & 0.88 & \\
\hline Atrial fibrillation & $113(7.7)$ & 28 & 0.74 & \\
\hline Atrial flutter & $13(0.9)$ & 4 & 0.68 & $<0.001$ \\
\hline Left ventricular hypert & ed by ECG) & & & \\
\hline Yes & 1021 (68.9) & 132 & 0.86 & \\
\hline No & $461(31.1)$ & 61 & 0.87 & 0.814 \\
\hline LBBB & & & & \\
\hline Yes & $45(3.3)$ & 15 & 0.66 & \\
\hline No & 1303 (96.7) & 165 & 0.87 & $<0.001$ \\
\hline Apical hypertrophy & & & & \\
\hline Yes & $532(41.1)$ & 56 & 0.89 & \\
\hline No & 763 (58.9) & 110 & 0.85 & 0.031 \\
\hline CTR (\%) & & & & \\
\hline$<50$ & $353(26.7)$ & 25 & 0.93 & \\
\hline $50-54$ & $484(36.7)$ & 54 & 0.89 & \\
\hline $55-59$ & $283(21.4)$ & 36 & 0.87 & \\
\hline$\geqslant 60$ & 200 (15.2) & 59 & 0.70 & $<0.001$ \\
\hline LVEF (\%) & & & & \\
\hline$<50$ & $67(5.9)$ & 27 & 0.59 & \\
\hline $50-59$ & $88(7.8)$ & 19 & 0.77 & \\
\hline $60-69$ & $243(21.5)$ & 29 & 0.88 & \\
\hline 70-79 & $439(38.9)$ & 49 & 0.89 & \\
\hline$\geqslant 80$ & $292(25.9)$ & 31 & 0.89 & $<0.001$ \\
\hline Thickness of IVS (mm) & & & & \\
\hline$<11$ & $145(9.8)$ & 17 & 0.88 & \\
\hline $11-15$ & $540(36.5)$ & 62 & 0.88 & \\
\hline $16-20$ & $476(32.1)$ & 68 & 0.85 & \\
\hline $21-25$ & $208(14.0)$ & 37 & 0.83 & \\
\hline$\geqslant 26$ & $112(7.6)$ & 18 & 0.83 & 0.255 \\
\hline Hospital beds & & & & \\
\hline$<299$ & $140(8.9)$ & 27 & 0.79 & \\
\hline $300-399$ & $93(5.9)$ & 19 & 0.80 & \\
\hline $400-499$ & $121(7.7)$ & 14 & 0.88 & \\
\hline$\geqslant 500$ & $360(22.8)$ & 60 & 0.83 & \\
\hline University hospital & $867(54.8)$ & 93 & 0.89 & 0.002 \\
\hline Time from diagnosis & & & & \\
\hline$<1$ & $463(30.6)$ & 62 & 0.86 & \\
\hline $1-1.9$ & $116(7.7)$ & 16 & 0.85 & \\
\hline $2-2.9$ & $144(9.5)$ & 15 & 0.89 & \\
\hline $3-3.9$ & $97(6.4)$ & 14 & 0.85 & \\
\hline $4-4.9$ & $97(6.4)$ & 14 & 0.85 & \\
\hline $5-9.9$ & $312(20.6)$ & 35 & 0.89 & \\
\hline$\geqslant 10$ & $283(18.7)$ & 51 & 0.81 & 0.231 \\
\hline
\end{tabular}

BMI, body mass index; CTR, cardiothoracic ratio; HCM, hypertrophic cardiomyopathy; IVS, interventricular septum; LBBB, left bundle branch block; LVEF, left ventricular ejection fraction; NYHA, New York Heart Association. 
Table 2 Multivariate-adjusted HRs of predictive variables for all-cause mortality in patients with hypertrophic cardiomyopathy

\begin{tabular}{|c|c|c|c|}
\hline & $\begin{array}{l}\text { Adjusted HR* } \\
(95 \% \mathrm{CI})\end{array}$ & $\begin{array}{l}\text { Wald } \\
\text { statistic }\end{array}$ & p Value \\
\hline \multicolumn{4}{|l|}{ Sex } \\
\hline Men & 1 & & \\
\hline Women & $0.77(0.46$ to 1.30$)$ & 1 & 0.324 \\
\hline \multicolumn{4}{|l|}{ Age } \\
\hline 1-year increase & 1.02 (1.01 to 1.04$)$ & 8.2 & 0.004 \\
\hline \multicolumn{4}{|l|}{ Diabetes mellitus } \\
\hline Yes & $1.03(0.47$ to 2.28$)$ & $<0.1$ & 0.941 \\
\hline No & 1 & & \\
\hline \multicolumn{4}{|l|}{ NYHA classification } \\
\hline 1 & 1 & & \\
\hline$\|$ & $1.19(0.74$ to 1.94$)$ & 0.5 & 0.472 \\
\hline III & 3.41 (1.75 to 6.67$)$ & 12.9 & $<0.001$ \\
\hline IV & $2.85(0.87$ to 9.39$)$ & 3.0 & 0.084 \\
\hline \multicolumn{4}{|l|}{ Rhythm } \\
\hline Sinus rhythm & 1 & & \\
\hline Atrial fibrillation & $1.36(0.75$ to 2.46$)$ & 1.0 & 0.306 \\
\hline Atrial flutter & $0.62(0.16$ to 2.30$)$ & 0.5 & 0.471 \\
\hline \multicolumn{4}{|l|}{ LBBB } \\
\hline Yes & 3.14 (1.28 to 7.71$)$ & 6.2 & 0.013 \\
\hline No & 1 & & \\
\hline \multicolumn{4}{|l|}{ Apical hypertrophy } \\
\hline Yes & $0.58(0.36$ to 0.92$)$ & 5.4 & 0.021 \\
\hline No & 1 & & \\
\hline CTR† (increase of $6.2 \%$ ) & $1.61(1.26$ to 2.05$)$ & 14.4 & $<0.001$ \\
\hline BMI† (increase of $3.4 \mathrm{~kg} / \mathrm{m}^{2}$ ) & $0.80(0.63$ to 1.01$)$ & 3.7 & 0.056 \\
\hline $\begin{array}{l}\text { Thickness of IVS† (increase } \\
\text { of } 5.8 \mathrm{~mm} \text { ) }\end{array}$ & $1.15(0.94$ to 1.40$)$ & 1.9 & 0.163 \\
\hline LVEF (decrease of 13\%) & $1.42(1.20$ to 1.69$)$ & 16.4 & $<0.001$ \\
\hline
\end{tabular}

BMI, body mass index; CTR, cardiothoracic ratio; IVS, interventricular septum; LBBB, left bundle branch block; LVEF, left ventricular ejection fraction; NYHA, New York Heart Association.

*All variables are included in the same model.

tHazard ratio for 1 SD increase or decrease; value of 1 SD given in parentheses.

\section{DISCUSSION}

In this paper, we report the crude probability of death and the HRs for all-cause mortality by baseline prognostic factors, from a nationwide study of HCM in Japan. To our knowledge, this is the first nationwide follow-up survey in Japan conducted on patients with HCM.

One methodological issue in this survey involves the diagnostic criteria used. In 1995, the World Health Organization/International Society and Federation of Cardiology task force reported a new definition and classification of cardiomyopathy in which the cardiomyopathies were defined simply as diseases of the myocardium associated with cardiac dysfunction. ${ }^{11}$ However, we used the definition and classification provided by the earlier task force of $1980,{ }^{9}{ }^{10}$ in which idiopathic cardiomyopathy was distinguished from other specific heart muscle diseases. Our reasons for doing this were, firstly, that nearly all cardiologists and specialists in general medicine in Japan have been applying this definition to their diagnosis of cardiomyopathies for a long time; and secondly, that numerous previous reports have also used the same definition, allowing us to compare our data with those reports.

\section{General clinical outcome}

Several previous clinical studies on the natural history and prognosis of HCM have been based on populations of selected patients from referral centres, ${ }^{12-14}$ and therefore, on the basis of different levels of care and management, various prognoses would be expected. The clinical outcome and perception of prognostic factors in HCM is profoundly affected by a bias in patient selection. ${ }^{15}$ However, our study is free from this referral bias since patients were recruited from all over the nation and from different diagnostic centres.

The average (SD) age at entry into study was 58.0 (17.5) years. Both the overall crude probability of 5-year survival in our study $(86 \%)$ and the annual mortality $(2.2-$ $3.0 \%$ ) were comparable with the results from Western studies, ${ }^{14}{ }^{16}$ which were based on selected referral centres. According to previous report, ${ }^{8}$ the male/female ratio for HCM is $2: 3$ in Japan. In our study, the probability of survival did not differ between women and men, a finding which is consistent with previous data on sex comparisons of survival in patients with HCM. ${ }^{16}$ However, in Chinese patients, HCM was found to have a worse clinical outcome in female patients. ${ }^{13}$ The reason for this inconsistency between our data and Chinese patients is not clear, but the small number of participants in the Chinese study and the point that less female than male patients were in the stage I of the NYHA function class in their study may explain the difference with our result.

\section{Prognostic factors}

Our results suggest for the first time that a simple chest $x$ ray could be a reliable prognostic predictor in HCM. Although a CTR of $55-59 \%$ ( $21.4 \%$ of study patients) posed a slight increase in the risk of mortality, a CTR of $>60 \%$ (15.2\% of study patients) was strongly associated with a poorer prognosis. LVEF at baseline was also found to be a significant predictor of allcause mortality in our study, with an LVEF of $<60 \%$ (13.7\% of study patients) associated with poorer prognosis. Progression of HCM to LV dilatation and systolic dysfunction sometimes occurs, although the mechanism of this is not fully understood ${ }^{17}$ However, data are limited on the prognosis of different LVEF levels in patients with HCM. Our findings confirmed the result of a previous study involving 10 patients with HCM, which concluded that an LVEF of $<50 \%$ was associated with poor prognosis. ${ }^{18}$

Apical hypertrophy has been associated with a more benign prognosis and rarely with cardiovascular mortality and morbidity in Western populations of patients with HCM. ${ }^{19}{ }^{20}$ Our data support this finding, as patients with apical hypertrophy had a better prognosis in the multivariate model. Previous studies in the Japanese population have also indicated a benign prognosis in patients with this condition..$^{21} 22$

A direct relationship between LV wall thickness and the risk of sudden death or heart failure-related death has been reported in patients with HCM. ${ }^{23-25}$ Our data did not support this finding, as LV wall thickness did not show a significant relationship with prognosis. Our results support previous findings that suggest LV wall thickness should not be considered as an isolated risk factor for mortality due to cardiovascular diseases in patients with HCM. ${ }^{26}{ }^{27}$ Olivotto et al ${ }^{27}$ proposed that the presence of LV hypertrophy might be a potential risk factor for sudden death only in those patients diagnosed with HCM at a very young age.

In a community-based HCM study, AF was reported to be a substantial risk factor for heart failure-related mortality and severe functional disability. ${ }^{28}$ The prevalence of AF was lower ( $7.7 \%$ of study patients) in our study than the $22 \%$ seen in this previous report. ${ }^{28}$ In our study, the crude survival rate for allcause mortality in patients with AF at baseline was significantly lower than for those patients with sinus rhythm. However, the significant relationship with AF disappeared in the multivariate model. We observed that the presence of LBBB at baseline was associated with a poorer prognosis on multivariate analysis. The infrequent occurrence of LBBB in our study (3.3\%) was comparable with previous data, which showed a prevalence of $6 \%$ among 204 patients with obstructive HCM and free from obstructive coronary artery disease. ${ }^{29}$ 


\section{Study limitations}

Although the follow-up time in our study was limited to 5 years, shorter than follow-up times in previous studies, ${ }^{13} 2027$ our study group was an average of 10 times larger than that of those studies, thus covering a comparable number of patientyears for the subgroups of baseline prognostic factors. A limitation of this study, which is shared by other studies, ${ }^{13}{ }^{26}$ is that a single measurement of prognostic factors, although reproducible and practical for clinical purposes, does not accurately reflect the total burden of predictors in individual patients. Another limitation of this study is that we failed to differentiate HCM-related deaths including sudden deaths and deaths caused by end-stage cardiac failure from other causes of mortality, and we reported all-cause mortality as our main outcome.

\section{CONCLUSIONS}

HCM has relatively good prognosis in Japanese patients. A poorer prognosis in HCM is predicted by high cardiothoracic ratio, low LVEF and the presence of LBBB, with a better prognosis in patients with apical hypertrophy. The presence of hypertension, AF and the level of IVS thickness were not independent predictors of prognosis during the 5-year followup period.

This study was supported by a grant-in-aid for the Epidemiology of Intractable Diseases Research Committee and a grant-in-aid for Idiopathic Cardiomyopathy Research Committee from the Ministry of Health and Welfare of Japan.

\section{Authors' affiliations}

Ali Nasermoaddeli, Katsuyuki Miura, Yoshiyuki Soyama, Yuko Morikawa, Hideaki Nakagawa, Department of Epidemiology and Public Health, Kanazawa Medical University, Ishikawa, Japan

Akira Matsumori, Department of Cardiovascular Medicine, Kyoto University Graduate School of Medicine, Kyoto, Japan

Akira Kitabatake, Department of Cardiovascular Medicine, Hokkaido University Graduate School of Medicine, Sapporo, Japan

Yutaka Inaba, Department of Epidemiology and Environmental Health, Juntendo University School of Medicine, Tokyo, Japan

Competing interests: None declared.

We disclose that there are no other financial, personal or professional relationships with other people or organisations that could be perceived as conflicts of interest, or as potentially influencing or biasing this work.

\section{REFERENCES}

1 Maron BJ. Hypertrophic cardiomyopathy: a systematic review. JAMA 2005;287:1308-20

2 Maron BJ. Hypertrophic cardiomyopathy. Lancet 1997;350:127-33.

3 Spirito $\mathbf{P}$, Seidman CE, Mckenna WJ, et al. Management of hypertrophic cardiomyopathy. N Engl J Med 1997:30:775-85.

4 Wigle ED, Rakowski H, Kimball BP, et al. Hypertrophic cardiomyopathy: clinical spectrum and treatment. Circulation 1995;92:1680-92.

5 Louie EK, Edwards LC. Hypertrophic cardiomyopathy. Prog Cardiovasc Dis 1994;36:275-308.
6 Maron BJ. Hypertrophic cardiomyopathy: an important global disease. Am J Med 2004;116:63-6.

7 Matsumori A, Furukawa Y, Hasegawa K, et al. Epidemiological and clinical characteristics of cardiomyopathies in Japan: results from nationwide surveys. Circ J 2002;66:323-36.

8 Miura K, Nakagawa H, Morikawa Y, et al. Epidemiology of idiopathic cardiomyopathy in Japan: results from a nationwide survey. Heart 2002:87:126-30

9 The WHO/ISFC task force on the definition and classification of cardiomyopathies. Report of the WHO/ISFC task force on the definition and classification of cardiomyopathies. Br Heart J 1980;44:672-3.

10 Research committee on idiopathic cardiomyopathy. Guidelines for the diagnosis of idiopathic cardiomyopathy. In: Annual report of the research committee on idiopathic cardiomyopathy [in Japanese]. Tokyo: Ministry of Health and Welfare, Japan, 1985:13-15.

11 Richardson P, Mckenna W, Bristow M, et al. Report of the 1995 World Health Organization/International Society and Federation of Cardiology Task Force on the definition and classification of cardiomyopathies. Circulation 1996;93:841-2.

12 Takagi E, Yamakado T, Nakano T. Prognosis of completely asymptomatic adult patients with hypertrophic cardiomyopathy. J Am Coll Cardiol 1999;33:206-11.

$13 \mathrm{Ho} \mathrm{HH}$, Lee KL, Lau CP, et al. Clinical characteristics of long-term outcome in Chinese patients with hypertrophic cardiomyopathy. Am J Med 2004;116:19-23.

14 Maron BJ, Casey SA, Hauser RG, et al. Clinical course of hypertrophic cardiomyopathy with survival to advanced age. J Am Coll Cardiol 2003;42:882-8.

15 Maron BJ, Spirito P. Impact of patient selection biases on the perception of hypertrophic cardiomyopathy and its natural history. Am J Cardiol 1993;72:970-2.

16 Dimitrow PP, Czarnecka D, Kawecka-Jaszcz k, et al. Sex-based comparison of survival in referred patients with hypertrophic cardiomyopathy. Am J Med 2004;117:65-6.

17 Nakamura K, Kusano KF, Matsubara H, et al. Relationship between oxidative stress and systolic dysfunction in patients with hypertrophic cardiomyopathy. J Card Fail 2005;11:117-23.

18 Doi K, Toda G, Iliev II, et al. Clinical analysis of hypertrophic cardiomyopathy which evolved into dilated phase during long-term follow-up. Jpn Heart $J$ 1999;40:579-87.

19 Webb JG, Sasson Z, Rakowski H, et al. Apical hypertrophic cardiomyopathy: clinical follow-up and diagnosis correlates. J Am Coll Cardiol 1990;15:83-90.

20 Eriksson MJ, Sonnenberg B, Woo A, et al. Long term outcome in patients with apical hypertrophic cardiomyopathy. J Am Coll Cardiol 2002;39:638-45.

21 Sakamoto T, Amano K, Hada Y, et al. Asymmetric apical hypertrophy: ten years experience. Postgrad Med J 1986;62:567-70.

22 Sakamoto T, Suzuki J. Apical hypertrophic cardiomyopathy. Nippon Rinsho 2000;58:93-101.

23 Maron BJ, Casey SA, Poliac LC, et al. Clinical course and prognosis of hypertrophic cardiomyopathy in a general United States cohort. JAMA 1999;281:650-5.

24 Spirito P, Maron BJ. Relation between extent of left ventricular hypertrophy and occurrence of sudden cardiac death in hypertrophic cardiomyopathy. J Am Coll Cardiol 1990;15:1521-6.

25 Spirito $\mathrm{P}$, Bellone $\mathrm{P}$, Harris KM, et al. Magnitude of left ventricular hypertrophy and risk of sudden death in hypertrophic cardiomyopathy. N Engl J Med 2000:342:1778-85.

26 Elliott PM, Gimeno-Blanes JR, Mahon NG, et al. Relation between severity of leftventricular hypertrophy and prognosis in patients with hypertrophic cardiomyopathy. Lancet 2001:357:420-4.

27 Olivotto I, Gistri R, Petrone P, et al. Maximum left ventricular thickness and risk of sudden death in patients with hypertrophic cardiomyopathy. J Am Coll Cardiol 2003;41:315-21

28 Olivotto I, Cecchi F, CaseySA, et al. Impact of atrial fibrillation on the clinical course of hypertrophic cardiomyopathy. Circulation 2001;104:2517-24.

29 Qin JX, Shiota T, Lever HM, et al. Conduction system abnormalities in patients with obstructive hypertrophic cardiomyopathy following septal reduction interventions. Am J Cardiol 2004;93:171-5. 